\section{DE LA DEMOCRATIZACIÓN DEL DEPORTE A LA HEGEMONÍA DE LOS MERCADOS: EL CASO ESPAÑOL}

\author{
DEMOCRATIZATION OF SPORT TO THE HEGEMONY OF MARKETS: \\ THE SPANISH CASE
}

\begin{abstract}
A DEMOCRATIZAÇÃO DO ESPORTE PARA A HEGEMONIA DOS MERCADOS: O CASO ESPANHOL
\end{abstract}

David Moscoso Sánchez*, Jesús Fernández Gavira*, Álvaro Rodríguez Díaz***
Palabras clave

Bienestar social. Políticas Públicas. Neoliberalismo. España.

Keywords

Social Welfare. Neoliberalism. Public policies. Spain.

Palavras-chave

Bem-estar social. Neoliberalismo. Políticas Públicas. Espanha.
Resumen: El sistema deportivo propio de la democracia está en crisis. Lo está porque el Estado de Bienestar, sobre el que se sustentan muchas de las políticas deportivas públicas, ha sido desbancado por un nuevo ciclo de hegemonía neoliberal. Para ilustrar esta realidad, nos centramos en el caso de España. En este país, se evidencia cómo la sociedad civil se encuentra poco representada por las decisiones políticas en materia deportiva, y cómo el deporte organizado ha dejado de cumplir una función social, al circunscribirse a los intereses de los agentes económicos. El artículo reclama de las ciencias sociales una postura crítica ante esta realidad.

Abstract: The sport system of democratic countries is in crisis. This is because the welfare state, on which many public sport policies are based, has been superseded by a new cycle of neoliberal hegemony. To illustrate this reality, we focus on the case of Spain. It becomes evident that civil society has a low representation in political decision making in sport matters and that organized sport has failed to fulfill a social function, being confined to the interests of economic agents. This article requests that social sciences take a critical stance with regards to this reality.

Resumo: 0 sistema esportivo próprio da democracia está em crise. Isto porque o Estado de bem-estar, sobre o qual se sustentam muitas das políticas públicas ao esporte, tem sido desbancado por um novo ciclo de hegemonia neoliberal. Para ilustrar essa realidade, nos centramos no caso espanhol. Evidencia-se como a sociedade civil se encontra pouco representada pelas decisões políticas em matéria esportiva, e como 0 esporte organizado tem deixado de cumprir uma função social, ao circunscrever-se aos interesses dos agentes econômicos. $\mathrm{O}$ artigo reclama das ciências sociais uma postura crítica ante tal realidade.
*Pablo de Olavide University. Sevilla, España. E-mail:dmoscoso@upo.es

${ }^{* *}$ Faculty of Educational Sciences. University of Seville. Sevilla, España.Email: jesusfgavira@us.es

*** Faculty of Educational Sciences. University of Seville. Sevilla, España.Email: jalvaro@us.es

Recebido em: 29-05-2014 Aprovado em: 23-09-2014

(c) (1) () Licence 


\section{INTRODUCCIÓN}

El deporte constituye un buen observatorio desde el que analizar el proceso de crisis del Estado de Bienestar. La idea del Estado de Bienestar o Sociedad del Bienestar se convirtió en el emblema ideológico (el consenso keynesiano frente al laissez faire liberal) de Europa al finalizar la Segunda Guerra Mundial, y ha marcado el devenir histórico de esta región hasta tiempos recientes. La reconstrucción de los Estados europeos desde la convicción de que éstos debían ser garantes del acceso de la ciudadanía a la sanidad, la educación o el trabajo, entre otros servicios, hizo que desde entonces también el deporte se encontrara marcado por esta lógica.

En su plasmación europea, en las últimas décadas del siglo XX el deporte ha sido objeto de numerosas políticas públicas que han contribuido a promocionarlo en todos los estratos de la sociedad. El empeño por garantizar la presencia del deporte en nuestras vidas como derecho ciudadano se remonta a la constitución del Tratado de Roma en 1957. Con su impulso, el Consejo de Europa inició una intensa actividad encaminada a la promoción del deporte, su tipificación en el sistema educativo y la regulación del deporte reglado. La Carta Europea del Deporte para Todos de 1975 (COMISIÓN EUROPEA, 1976, p. 2) constituye el mejor ejemplo de este afán potenciador del deporte en la sociedad europea. En ella se sostenía que:

[...] la promoción del deporte, como factor importante del desarrollo humano, debe ser estimulada y sostenida de forma adecuada por los fondos públicos, cualquiera que sea su edad, sexo, profesión, a comprender el valor del deporte y a practicarlo durante toda la vida, [lo que obliga a las Administraciones Públicas a garantizar] mejores condiciones para la práctica del deporte para todos, dejando de ser privilegio de minorías para pasar a ser un logro y una necesidad de toda sociedad.

Y esta idea ha adquirido fuerza de forma progresiva, manifestándose en los últimos años con mayor visibilidad a través de expresiones como una renovada Carta Europea del Deporte de 1992 y el Libro Blanco sobre el Deporte (COMISIÓN EUROPEA, 2007, p. 2), donde se recoge que:

[...] el deporte es un fenómeno social y económico que cada vez más contribuye significativamente al logro de los objetivos estratégicos de solidaridad y prosperidad de la Unión Europea. El deporte genera importantes valores como el trabajo en equipo, la solidaridad, la tolerancia y la lealtad. Promueve la participación activa de los ciudadanos de la Unión Europea. La Comisión reconoce el importante papel del deporte en la sociedad europea.

En este marco, la sociedad civil europea ha dinamizado la actividad deportiva durante décadas. Al fin y al cabo, la democratización implícita en la teoría del Estado de Bienestar no es más que un proceso de conversión civil del propio Estado; en última instancia, éste hace recaer en la sociedad los medios para responder a sus propias demandas civiles (GIL-CALVO, 1995).

En el caso del deporte, esta idea se plasmó en la actividad desarrollada a través de los clubes y federaciones deportivas, que resultó decisiva para su progresiva institucionalización (figura 1). Bajo el paraguas del Estado de Bienestar y del sistema democrático, la ciudadanía pudo organizarse de forma autónoma para promocionar la cultura deportiva. Este esfuerzo ha contribuido en estas décadas a que en muchos países de la Unión Europea la práctica deportiva se multiplicase y llegase a todos los estratos de la sociedad. 


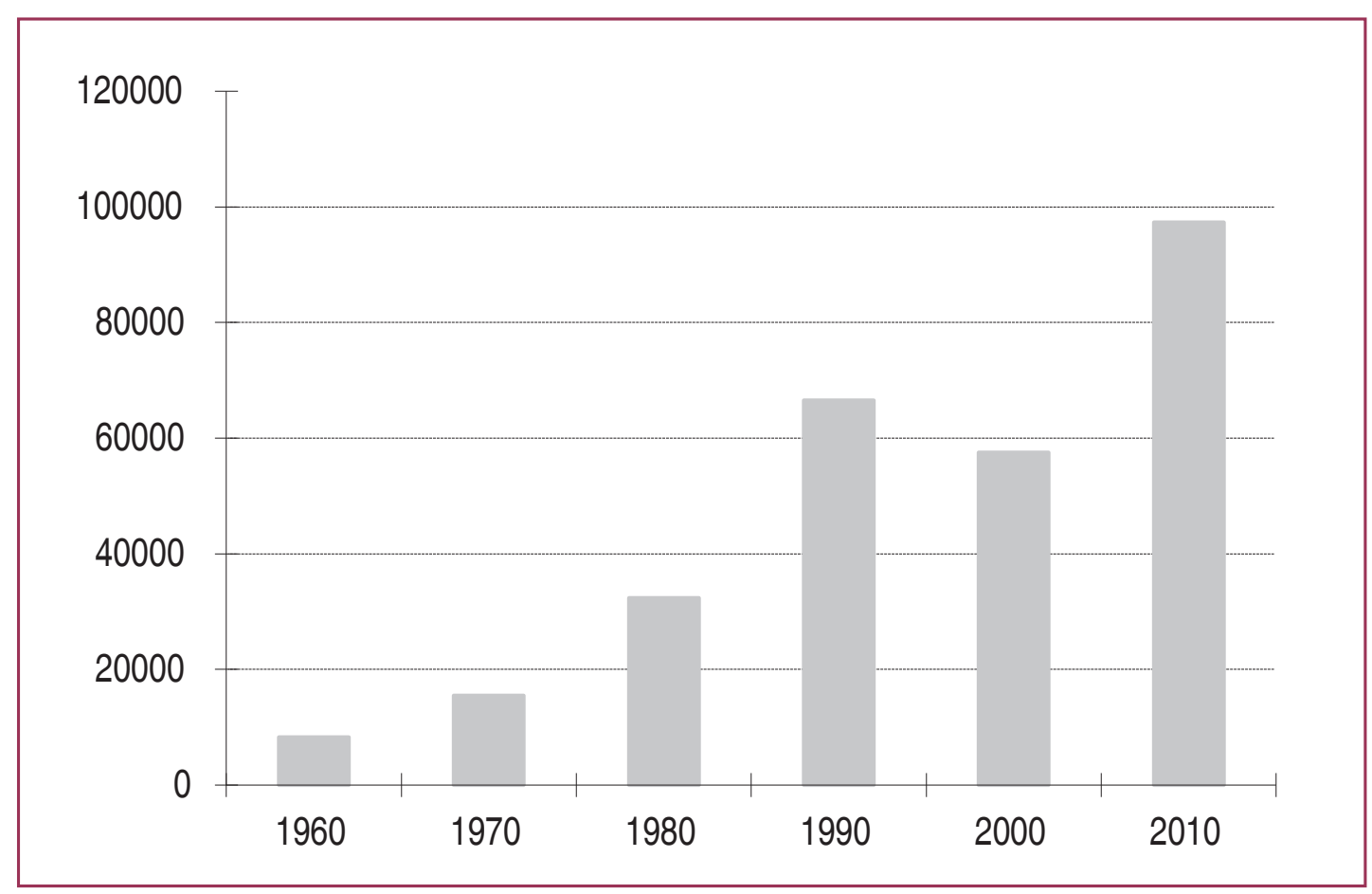

Fuente: INSTITUTO NACIONAL DE ESTADÍSTICA, 2014.

Las propias estadísticas oficiales ponen de manifiesto que en 1975 en países como España existían pocas instalaciones deportivas (0,5 instalaciones por cada mil practicantes), había menos personas que podían acceder a éstas (el 53\% eran de naturaleza privada) y, además, había pocos practicantes de deporte (22\% de la población), fundamentalmente las clases sociales medias-altas.

Desde el proceso de instauración del Estado de Bienestar en España, el deporte ha logrado conquistar el espacio público de la ciudadanía y convertirse en un ámbito con gran influencia social, política y económica. Sin embargo, en el plano de la realidad, tal como también se señala en el Libro Blanco sobre el Deporte (2007, p. 2), esta actividad:

[... s se enfrenta a nuevas amenazas y problemas que surgieron en la sociedad europea, como la presión comercial, la explotación de jóvenes deportistas, el dopaje, el racismo, la violencia, la corrupción y el lavado de dinero.

Ciertamente, en los años noventa del pasado siglo se produjo un cambio en el sistema deportivo europeo. Algunos autores han señalado al respecto su preocupación por el peso que las grandes fuerzas económicas han tenido en la mercantilización del deporte, y sobre los perjuicios que ello ha deparado para el desempeño de la función pública del deporte (ROBERTSON; GIULIANOTTI, 2006). Esto hace que, frente a la retórica política e institucional que ha envuelto en las décadas precedentes muchas de las políticas deportivas de Europa, en la actualidad su sistema deportivo se ha resentido como servicio público y ha depositado el peso de las grandes decisiones que marcan su devenir en los agentes económicos (mass media, multinacionales, marcas deportivas y sector financiero).

Quizá esto explique el que la práctica del deporte en la mayoría de los países de la Unión Europea se haya estancado, pese al relevo generacional de una población instruida en la cultura deportiva. Además, persiste lo que Moscoso (2013) ha denominado "brecha deportiva", 
refiriéndose al hecho de que aún siguen siendo numerosas las personas que no realizan esta actividad por razones culturales y económicas.

No obstante lo anterior, hay que matizar que este proceso se ha visto acompañado de una socialización de la ideología neoliberal en la propia cultura de la ciudadanía, que ha propiciado una acomodación de la situación descrita. No en vano, apenas hemos asistido hasta tiempos recientes a algún tipo de movilización social en contra de esta hegemonía de los mercados sobre las decisiones políticas en materia deportiva. Las críticas de la opinión pública en España en los últimos años contra la candidatura olímpica Madrid 2020, o las expresadas ante las primas económicas que el Estado español ofrecía a los jugadores de la Selección Española ante una hipotética victoria en la Copa del Mundo de Fútbol Brasil 2014, ponen en evidencia una emergente toma de conciencia pública sobre la política deportiva predominante, pero constituyen a la vez un indicador de la asimilación de estos valores neoliberales en la propia ciudadanía, al no haber existido crítica antes. Esta ambivalencia que sólo parece reciente en el deporte, al menos en España, forma parte desde antaño del sistema de valores de nuestra sociedad, tal como han mostrado algunos autores (BELL, 1977; INGLEHART, 1991; BECK; GIDDENS; LASH, 1997).

Este artículo trata de dilucidar esta realidad paradójica del deporte contemporáneo. De difundirse en Europa y en España bajo el paraguas de la convincente ideología del Estado de Bienestar y el sistema democrático, ha acabado lastrado por las perversiones propias del neoliberalismo. Y ello ha terminado por imponerse en la realidad social, las instituciones y las políticas de los tiempos actuales.

Para ilustrar este proceso, nos hemos centrado en el caso de España; un país en el que el deporte adquirió una importancia sin precedentes a lo largo de los años ochenta y donde, tras la celebración de los Juegos Olímpicos de Barcelona 92, despertó una inusitada pasión en la ciudadanía. La realidad de esa tendencia de crisis del Estado de Bienestar y de hegemonía de los mercados en el sistema deportivo en Europa se expresa con gran claridad en este país. De una parte, su ciudadanía se siente apabullada por la influencia de sus deportistas y las victorias de sus equipos nacionales. Pero, de otra parte, casi cuatro décadas más tarde de la experiencia democrática y de Estado de Bienestar, sigue siendo escaso el porcentaje de población que practica deporte con asiduidad (apenas 2 de cada 10); continúan persistiendo las barreras y desigualdades sociales en el acceso a la práctica del deporte (manteniéndose bajos niveles de práctica entre mujeres, mayores y ciudadanos con bajos niveles educativos e ingresos económicos); han crecido los delitos relacionados con el dopaje y el tráfico ilegal de substancias dopantes (por ejemplo, la "Operación Puerto" que implicó a la atleta olímpica Marta Domínguez, o la "Operación Galgo"); afloran los escándalos públicos por corrupción (por ejemplo, los casos Palma Arena, Bernabeu o Noos) y de evasión fiscal (por ejemplo, la denuncia de la Agencia Tributaria contra futbolistas como Messi o Neymar); y, por último, la gestión de los eventos y las agendas deportivas del país se encuentran en manos de los medios de comunicación y no de la Administración del Estado.

La hipótesis que manejamos gira sobre la idea de que, pese al esfuerzo del Estado de Bienestar, el deporte en España como derecho ciudadano ha sido usurpado por el sistema neoliberal, y normalizado (de forma acrítica) como realidad en el seno de su sociedad. La revisión de la agenda política - y, con ella, de los procesos, los hitos y los cambios sociales - que ha trazado los casi cuarenta años de democracia en España y el 
análisis de las principales estadísticas oficiales del deporte en este país, nos permiten vislumbrar este proceso de apropiación del deporte como servicio público por parte del poder privado. Somos conscientes de la pluralidad de enfoques teóricos desde los que se puede abordar este análisis. Nosotros nos postulamos por un enfoque crítico, próximo al legado de autores como Brohm (1982), que han marcado una tradición en el estudio del deporte desde el marxismo.

\section{FUENTES DE INFORMACIÓN}

El presente trabajo se basa en la explotación de datos secundarios. Se apoya en las dos principales fuentes de referencia estadística y sociológica del deporte en España: la Encuesta de Hábitos Deportivos de los Españoles y el Censo Nacional de Instalaciones Deportivas.

La Encuesta de Hábitos Deportivos de los Españoles surge tras la aplicación de la Ley 13/1980 General de la Cultura Física y del Deporte (ESPAÑA, 1980). En los inicios de la democracia en España, las administraciones públicas requerían datos para poder planificar adecuadamente las políticas deportivas. Por ese motivo, se creó una encuesta para conocer de forma comparada la evolución de las actitudes, los comportamientos y los hábitos deportivos de los españoles (MOSCOSO; MUÑOZ; 2012). Ésta se ha venido realizando por el Centro de Investigaciones Sociológicas - a partir de ahora CIS - quinquenalmente entre 1980 y 2010. En este periodo se han llevado a cabo 7 encuestas (ver tabla 1).

Tabla 1: Serie histórica de la EHDE

\begin{tabular}{|c|c|c|}
\hline Encuestas & Muestra & Año \\
\hline Encuesta de Hábitos Deportivos nº.1257 & 4.493 & 1980 \\
\hline Encuesta de Hábitos Deportivos (sin $\left.n^{\circ}\right)^{*}$ & 1.557 & 1985 \\
\hline Encuesta de Hábitos Deportivos nº.2198 & 3.329 & 1990 \\
\hline Encuesta de Hábitos Deportivos n..2397 & 3.000 & 1995 \\
\hline Encuesta de Hábitos Deportivos n.2493 & 5.160 & 2000 \\
\hline Encuesta de Hábitos Deportivos n.2599 & 8.170 & 2005 \\
\hline Encuesta de Hábitos Deportivos nº.2833 & 8.925 & 2010 \\
\hline
\end{tabular}

Fuente: CENTRO DE INVESTIGACIONES SOCIOLÓGICAS, 2010..

*La encuesta de 1985 no la realizó el Centro de Investigaciones Sociológicas; la llevó a cabo el Consejo Superior de Deportes.

En lo que atañe al Censo Nacional de Instalaciones Deportivas - a partir de ahora CNID -, realizado por el Consejo Superior de Deportes - a partir de ahora CSD -, ofrece datos sobre el número y las características de las instalaciones deportivas españolas a lo largo de sus tres estudios censales (1986, 1997 y 2005) (tabla 2). Abarca las instalaciones deportivas situadas en 8.116 núcleos de población de 17 regiones españolas y 2 ciudades autónomas. Se han realizado 3 censos en este tiempo - actualmente se está culminando el último -. Éstos han permitido analizar la situación en materia de infraestructuras deportivas entre los distintos territorios españoles, pudiéndola comparar también desde una perspectiva temporal. Su pretensión es facilitar la puesta en práctica de los planes directores de infraestructuras deportivas en las distintas regiones. 
Tabla 2: Serie histórica del CNID

\begin{tabular}{lcc}
\hline \multicolumn{1}{c}{ Encuestas } & Muestra & Año \\
\hline I Censo Nacional de Instalaciones Deportivas & 4.493 & 1986 \\
II Censo Nacional de Instalaciones Deportivas & 1.557 & 1997 \\
III Censo Nacional de Instalaciones Deportivas & 8.925 & 2005 \\
\hline
\end{tabular}

Fuente: Elaboración propia a partir del CONSEJO SUPERIOR DE DEPORTES (2005, p 33).

Adicionalmente, nos hemos apoyado en los relatos producidos en el estudio de Moscoso, Martín, Sánchez y Pedrajas (2013), realizado para el Consejo Superior de Deportes del Gobierno de España (Ref.: 007/UPB10/12), con el fin de apoyar algunas de las hipótesis y argumentaciones sobre el objeto de este trabajo.

\section{3 ¿LA DEMOCRATIZACIÓN DEL DEPORTE EN ESPAÑA? DEL DEPORTE PARA TODOS AL MERCADO DEL DEPORTE}

Para comprender el proceso de usurpación del deporte como derecho ciudadano por parte de los mercados deportivos, así como el proceso de ideologización neoliberal del deporte en la superestructura, resulta útil revisar el proceso histórico vivido en la democratización del deporte en España.

\subsection{Los años gloriosos del deporte para todos}

García y Lagardera (1998) consideran que la transición democrática y, en particular, la Constitución Española de 1978, ejercieron un papel decisivo en la difusión del deporte, al ofrecer la oportunidad de que esta actividad dejara de ser un privilegio de las clases minoritarias. En el Capítulo Tercero del Título I de la Constitución (ESPAÑA, 1987), en su artículo 43.3 se señalaba que "[...] los poderes públicos fomentarán la educación sanitaria, la educación física y el deporte." Es posible compartir que, con este hito, la sociedad española al fin podría despojarse de las rémoras del pasado que le impedían acceder a la práctica del deporte. La democracia supuso un revulsivo en muchos campos sociales, inclusive el del deporte, que no se encontró ajeno a este clima de cambio y reivindicación. Tal como señalan García y Lagardera (1998, p. 37):

\footnotetext{
[...] se pretendía ampliar las bases del deporte popular, facilitar la práctica deportiva del mayor número posible de personas en instalaciones apropiadas; se aspiraba a universalizar la enseñanza y la práctica de la educación física y el deporte en la escuela, y todo ello desde la consideración del deporte como un derecho ciudadano, un servicio público.
}

A partir de ese momento, parece ser que se iría normalizando la situación deportiva en España, al oficializarse la enseñanza de la educación física y aumentar el número de instalaciones y practicantes.

La Constitución Española proveyó a las instituciones políticas y administrativas de una legislación básica para lograr este objetivo. La Ley 13/1980 General de la Cultura Física y el Deporte (ESPAÑA, 1980, p. 7909) pretendía, según su artículo 1, "[...] el impulso, la orientación y la coordinación de la educación física y del deporte, como factores imprescindibles en la formación y en el desarrollo integral de la persona."

La puesta en vigor de esta Ley significó, en un sentido práctico, que todos los centros educativos debían ofrecer unas infraestructuras deportivas suficientes para la enseñanza de la educación y la cultura física. Además, apoyaba la construcción de otros espacios e instalaciones 
deportivas públicas para la promoción del deporte. En esos años, España pasó de tener 19.418 instalaciones deportivas en 1975 a 48.723 en 1986 (un incremento del 60\%). En este último año, además, el 32\% de los centros educativos ya disponían de parte de esas instalaciones —en 2005 lo hacían el 79\%, según datos del III CNID (CONSEJO SUPERIOR DE DEPORTES, 2005).

En ese contexto, se da una coyuntura favorable para el definitivo impulso de la práctica del deporte en España: la aplicación de una serie de medidas relacionadas con los principios de la ya apuntada Carta Europea del Deporte para Todos de 1975. Ésta dirimía que la administración española debía promocionar la actividad deportiva y la construcción de instalaciones deportivas, a través de los ayuntamientos y los centros escolares (RODRÍGUEZ, 2008). La prueba de este impulso es que el número de practicantes de deporte aumentó un $11 \%$ desde 1980 a 1985 (del 25\% pasó al 34\%) (Figura 2), es decir, un crecimiento anual de casi un 2\% durante ese periodo.

Esta tendencia alcista del número de deportistas se mantuvo hasta mitad de los noventa del pasado siglo, de la misma manera que lo hizo el número de instalaciones deportivas. En 1995, el número de deportistas españoles rozaba el 39\%, según datos de la EHDE (CIS, 1995), y el número de instalaciones deportivas ascendía a 66.670 en 1997, según el II CNID (CSD, 1997) —un incremento del $27 \%$ con respecto a 1986 (CONSEJO SUPERIOR DE DEPORTES, 2005) (Figura 2).

A principios de los años noventa, la presencia del deporte había adquirido tal relevancia en la vida de los españoles que resulta difícil entender hoy a esta sociedad sin contemplar el deporte como una parte esencial de su cultura. Tal como expresó Lagardera (1992, p. 16):

[...] los hombres y mujeres de hoy han aprendido a vivir corriendo tras un balón, contemplando las finales olímpicas en el televisor, apostando dinero o utilizando los chándales y el calzado deportivo como indumentaria usual en sus prácticas físicas o en los descansos del fin de semana.

Con lo cual, más allá del incremento del número de deportistas, consideramos que lo importante es que el deporte acabó conquistando el imaginario cultural de los españoles.

Figura 2: Democratización del deporte en España (1975-2010). Hitos y etapas.

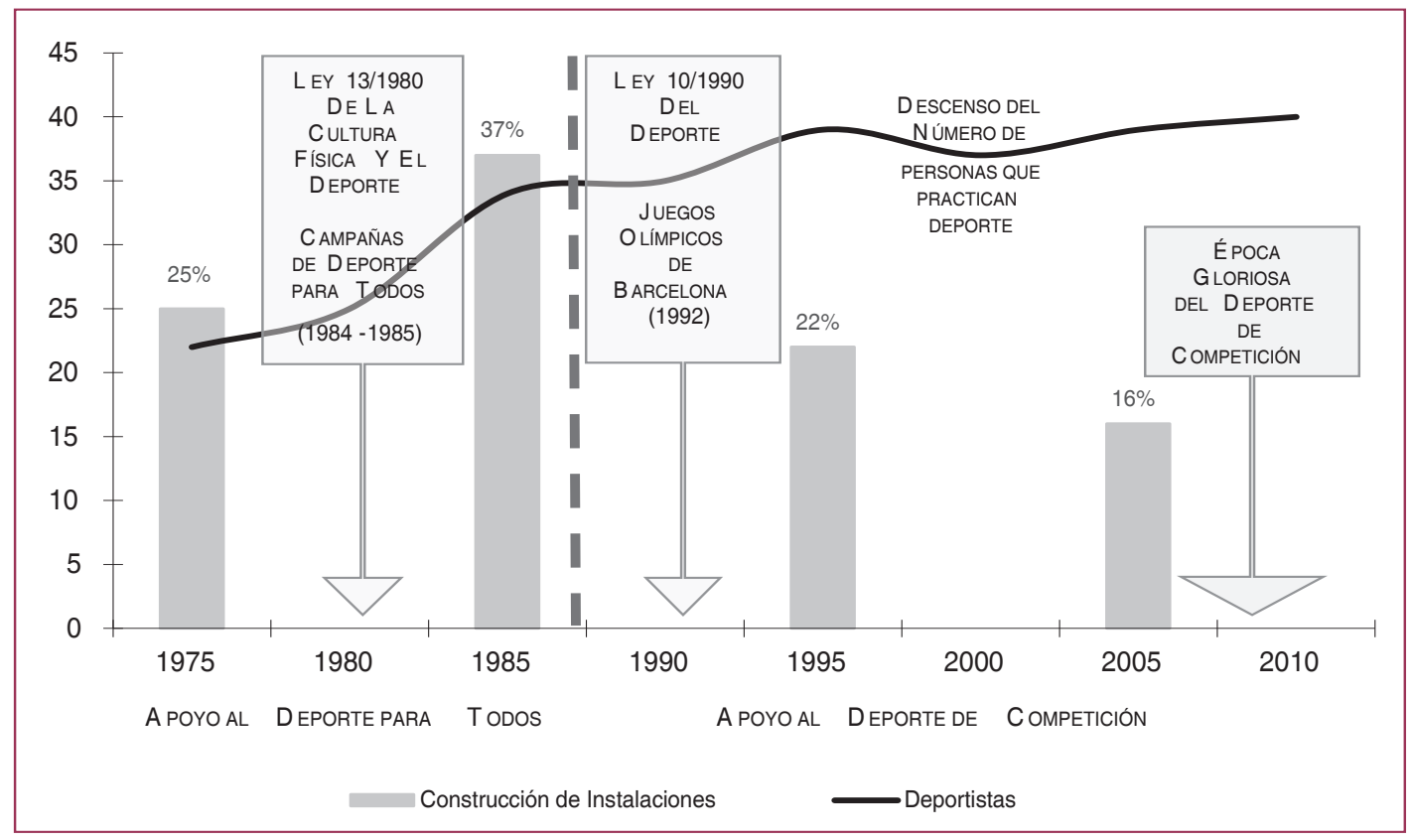

Fuente: Elaboración propia a partir de GARCÍA FERRANDO (1997) y CENTRO DE INVESTIGACIONES SOCIOLÓGICAS (1995, 2000, 2005 y 2010) (datos en porcentajes). 


\subsection{El dominio del deporte de competición y de espectáculo}

Esta tendencia prometedora que había marcado la democratización del deporte en España durante la primera década del periodo democrático pensamos que se disipó gradualmente. En primer lugar, el porcentaje de deportistas sólo ha crecido un 1\% entre 1995 y 2010 (un $39 \%$ y un $40 \%$, respectivamente) - contrastando con el crecimiento experimentado entre 1980 y 1995, que fue de un 11\%. En segundo lugar, el crecimiento del número de instalaciones deportivas construidas menguó considerablemente entre 1996 y 2005: si en 1996 la ratio de instalaciones deportivas por cada 1.000 habitantes era de 1,73, en 2010 era de 1,76, manteniéndose cifras parecidas (CONSEJO SUPERIOR DE DEPORTES, 2005).

Por todo ello, nosotros entendemos que la escasa reproducción de práctica deportiva está relacionada con un cambio en la política deportiva de España, que antepuso el deporte profesional y de espectáculo al deporte para todos. En realidad, nuestra opinión es que en ello influyeron los dos siguientes elementos: el cambio que se produce en la legislación que regula el deporte en España en 1990 y la celebración de los Juegos Olímpicos de Barcelona 92.

En cuanto al primer elemento, la Ley 13/1980 General de la Cultura Física y el Deporte queda derogada por un nuevo marco jurídico, la Ley 10/1990 del Deporte. Esta nueva Ley constituyó un punto de inflexión en la política deportiva española, por cuanto se marcó unas prioridades diferentes. La nueva Ley perseguía la regulación profesional del deporte y la consolidación del deporte de alta competición. En dicha Ley queda poco claro qué lugar ocupa el "deporte para todos". Con lo cual, si en el inicio de la democracia española el deporte emergió junto al concepto de ciudadanía en una simbiosis casi perfecta, predominando la idea del "deporte para todos", con la madurez alcanzada tras la puesta en vigor de la Ley 10/1990 del Deporte - que es la que sigue regulando la actividad deportiva en España veinticinco años después -, la ciudadanía queda relegada a un segundo plano, tras las organizaciones deportivas y el deporte profesional. Es eso lo que explica el cambio de tendencia en el ámbito del deporte español.

No es casual que esta Ley se creara dos años antes de los Juegos Olímpicos de Barcelona 92, y que desde entonces aumentaron los recursos públicos destinados al deporte de competición. En efecto, antes de la vigencia de la Ley 10/1990 del Deporte, el Gobierno de España asumió el apoyo a los deportistas de alto rendimiento a través de varias vías. Una de esas vías fue la puesta en marcha del Plan de Ayuda al Deporte Olímpico (ADO), en 1989, un programa específico para financiar la dedicación exclusiva al entrenamiento y la preparación de pruebas olímpicas de los deportistas de alto nivel. Otra de las vías de apoyo al deporte de alto nivel fue la dotación de instalaciones deportivas destinadas a la excelencia en el deporte, construyéndose 7 Centros de Alto Rendimiento y 36 Centros de Tecnificación Deportiva.

Tal como hemos constatado el aumento de los recursos públicos destinados al deporte de competición se viene produciendo en detrimento de los recursos para la educación física y el deporte para todos. Según datos extraídos del INE, la mayor parte del presupuesto público de la Administración del Estado español en materia deportiva - que apenas supone el 0,01\% del PIB nacional (3,3€ habitante/año) - está dirigido al deporte federado y al deporte de competición, el 92\% (136,4 millones de euros ${ }^{1}$, en una partida presupuestaria, y 3,2 millones de euros $^{2}$, en otra partida). Por el contrario, el presupuesto destinado a la promoción del deporte

1 Programa presupuestario 336A: Fomento y apoyo a las actividades deportivas. Fomento de la práctica deportiva federada, mejora de la gestión federativa, mejora de la alta competición, ordenación de la tecnificación y los títulos de educación física y deportiva, actividad de la Agencia Estatal Antidopaje.

2 Programa presupuestario 144A: Cooperación, promoción y difusión cultural en el exterior. Fomento de la actividad federativa en el exterior y potenciación de la presencia de España en organismos internacionales. 
para todos es irrelevante: el $8 \%$ del presupuesto en materia deportiva, y siempre tiene una carga competitiva (12,3 millones de euros 3 ) (figura 3 ).

Figura 3: Gasto público en deporte, según programación.

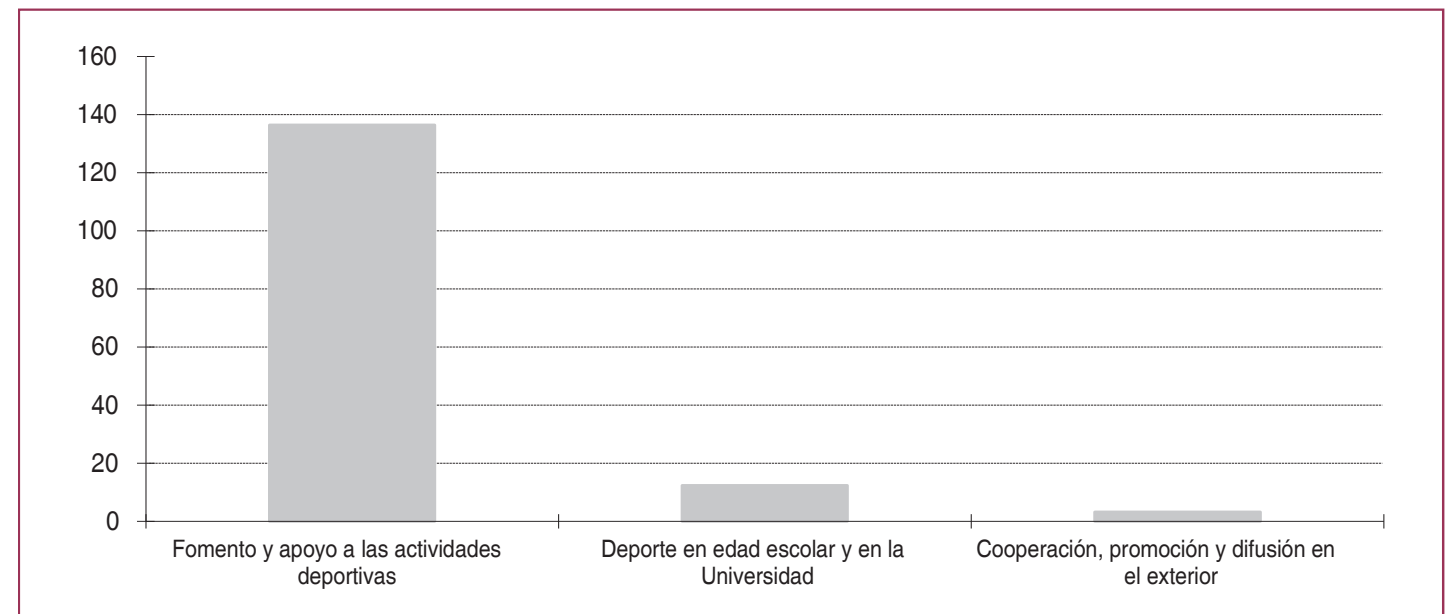

Fuente: MINISTERIO DE HACIENDA Y ADMINISTRACIONES PÚBLICAS. Liquidación de los Presupuestos del Estado. Ejercicio 2011 (datos en millones de $€$ )

En esta línea, puede comprobarse que la misma partida presupuestaria 322K: Deporte en Edad Escolar y en la Universidad, que mayormente está dirigida a organizar la competiciones escolares y universitarias, así como a mejorar los equipamientos deportivos en los centros educativos, movilizaron en el año 2012 a 11.109 niños y jóvenes, invirtiendo una media de $1.216 €$ por participante y año. Esta cifra parece irrelevante ante los elevados presupuestos del denominado Plan $\mathrm{ADO}^{4}$, del que disfrutaron en el periodo 2008-2012 alrededor de 370 deportistas, lo que supone una inversión de $42.567 €$ por deportista y año (figura 4).

Figura 4: Gasto público en deporte (Partida: $322 \mathrm{~K}$ : Deporte en edad escolar y en la Universidad.) y Financiación Plan ADO 2004-2008, 2008-2012 y 2012-2016.

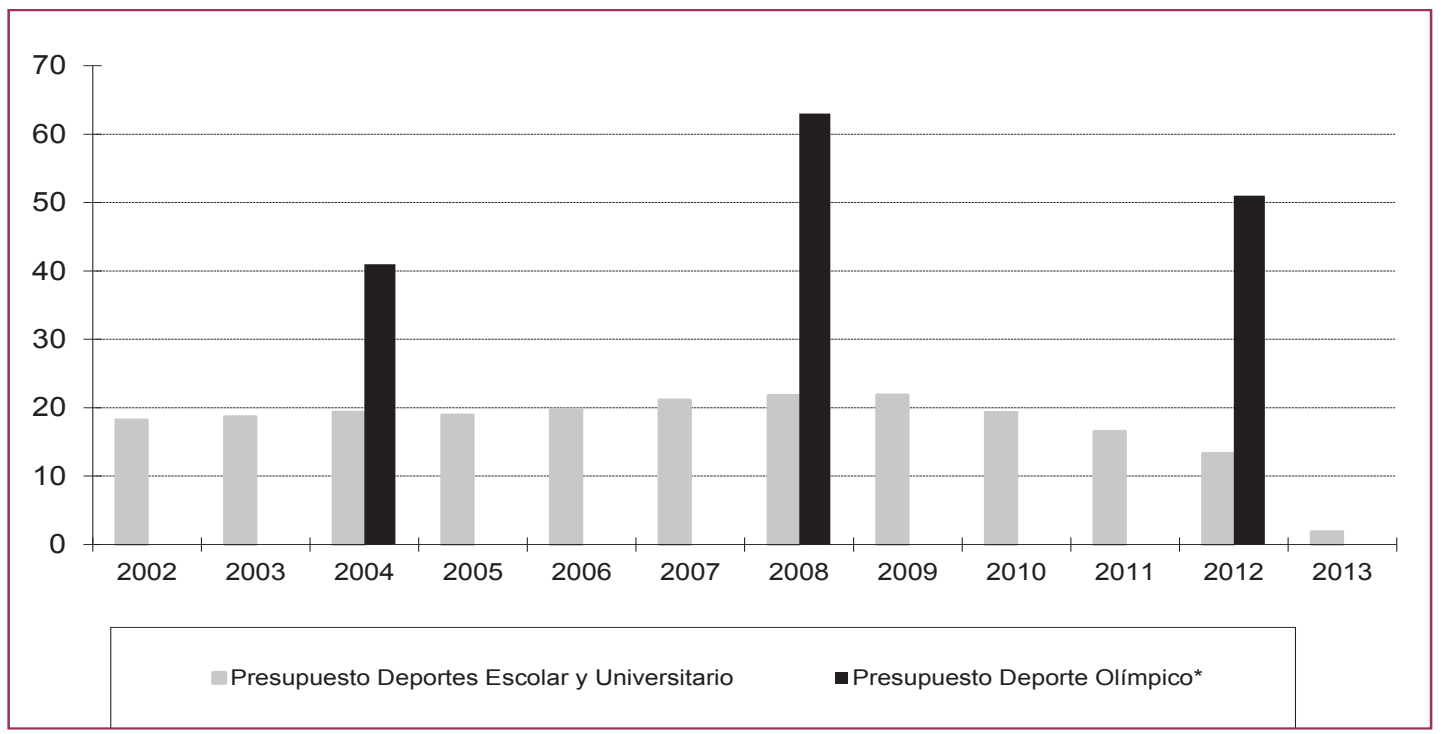

Fuente: MINISTERIO DE HACIENDA Y ADMINISTRACIONES PÚBLICAS.. Liquidación de los Presupuestos del Estado; y CONSEJO SUPERIOR DE DEPORTES (datos en millones de €).

3 Programa presupuestario 322K: Deporte en Edad Escolar y en la Universidad. Potenciar el deporte escolar y universitario en el ámbito nacional y potenciar las actuaciones de apoyo científico, investigación y desarrollo tecnológico para la investigación deportiva con fines aplicados.

4 Los presupuestos del Plan ADO tienen un carácter cuatrianual, de ahí que sólo se presenten en la figura 4 con esa periodicidad. La diferencia con respecto a los del deporte escolar no sólo radica en el volumen del presupuesto, sino en el número de personas que disfrutan de él. 
El hecho de que desde los años noventa a nuestros días los recursos destinados a la promoción del deporte para todos en España se hayan reducido significativamente, en favor de los destinados al deporte de competición, podría deberse, entre otros motivos, a que la competencia por promover el deporte para todos se ha ido transfiriendo de la Administración del Estado a los gobiernos regionales y desde éstos, a su vez, a los ayuntamientos, diluyéndose y limitándose la intervención pública en materia deportiva. Así, por ejemplo, en 2011, mientras que la Administración General del Estado dedicó el 0,07\% del gasto total de su presupuesto a las partidas en materia deportiva (3,3€ hab./año), los gobiernos regionales destinaron a estas partidas el $0,33 \%$ de sus presupuestos (12,6€ hab./año) y los ayuntamientos el 3,54\% (54,6€ hab./año) (tabla 3). Desde nuestro punto de vista, esta situación constituye un hándicap para los pequeños ayuntamientos, que se ven desvalidos de capacidad presupuestaria para promocionar el deporte para todos.

Tabla 3: Gasto público en deporte, según naturaleza de la administración.

\begin{tabular}{lccc}
\hline \multicolumn{1}{c}{$\begin{array}{c}\text { Gasto público } \\
\text { vinculado al deporte }\end{array}$} & $\begin{array}{c}\text { Administración } \\
\text { General del Estado }\end{array}$ & $\begin{array}{c}\text { Administraciones } \\
\text { Autonómicas }\end{array}$ & $\begin{array}{c}\text { Administraciones } \\
\text { locales }\end{array}$ \\
\hline$\%$ del gasto total & 0,07 & 0,33 & 3,52 \\
$\%$ del P.I.B. & 0,01 & 0,05 & 0,24 \\
Valores medios por habitante $(€)$ & 3,3 & 12,6 & 54,6 \\
\hline
\end{tabular}

Fuente: MINISTERIO DE HACIENDA Y ADMINISTRACIONES PÚBLICAS. Liquidación de los Presupuestos del Estado. Ejercicio 2011.

Este nuevo escenario podría explicarse, a su vez, entre otras razones, a tres diferentes circunstancias que escapan al ámbito estrictamente deportivo: el progresivo interés de los medios de comunicación de masas en los eventos deportivos, la mercadotecnia generada en torno al mundo del deporte y el cambio de valores experimentado en la sociedad española en estas tres décadas - al que ya nos referíamos en la introducción.

En relación a la primera circunstancia, en las últimas décadas los medios de comunicación han sabido transformar lo que era una simple actividad física y de disfrute colectivo en un producto de mercado audiovisual (DESBORDES; OHL; TRIBOU, 1999). Ello ha hecho del deporte un fenómeno de gran alcance. A este respecto, sólo en España 36,2 millones de telespectadores vieron la retransmisión de las principales competiciones celebradas durante los Juegos Olímpicos de Londres 2012; alrededor de 15 millones de telespectadores estuvieron ante la pantalla durante la final de la Copa del Mundo de Fútbol de Sudáfrica en 2010; y otros tantos hicieron lo propio en la final de la Copa del Rey jugada entre el Real Madrid y el Barcelona C.F. en 2011, según la EGM (2013). Ello supone un impacto mediático y un mercado audiovisual de indudable rédito. Ahora bien, lo cierto es que las características del fenómeno deportivo que se difunden a través de los mass media no están en sintonía con las motivaciones del deporte praxis entre una parte significativa de la ciudadanía, como pone en evidencia el trabajo de Moscoso et al. (2013).

Esta situación nos conduce a la segunda circunstancia, la mercadotécnica del deporte espectáculo, producida con las victorias, las noticias deportivas y las apuestas, el material deportivo, las esponsorizaciones, la publicidad, o las propias retransmisiones deportivas. Sólo en el ámbito del fútbol, según el Center for Sport Business Management, el impacto económico de este deporte en España genera 10.000 millones de euros al año, es decir, un 1\% del PIB. Y sólo por las retransmisiones, la Liga de Fútbol Profesional tiene unos compromisos de pago de 
600 millones de euros al año. Empresas como el grupo Mediapró tienen obligaciones de pago de 2.400 millones de euros por derechos deportivos, fundamentalmente con clubes de fútbol (GARCÍA; ALCOLEA, 2011). Como exponen Robertson y Giulianotti (2006), los presidentes de los grandes clubes de fútbol europeos son mayoritariamente magnates de la construcción o las finanzas, que, en connivencia con los grandes grupos de comunicación, se sirven de su posición para continuar gestando sus intereses empresariales.

La tercera circunstancia aludida tiene que ver con un aspecto de carácter transversal, que llega a todos los niveles de la cultura y la estructura social: el cambio en los valores de la ciudadanía española. En las últimas décadas se ha producido una difusión de los valores materialistas que afectan al deporte de la misma forma que otros ámbitos. Debido a la imagen proyectada por los mass media y las propias organizaciones deportivas en los últimos años, el deporte es percibido hoy como un medio de prestigio, enriquecimiento personal y de consumismo, lo cual es escenificado a través de la elite deportiva. Por ejemplo, la famosa plantilla de la "Era Galáctica" del Real Madrid, en las temporadas de 2000 a 2006, que imprimió a este deporte un cierto glamour, claramente expresaba esta imagen. Tanto es así que en España son muchos los padres y madres que expresan su deseo de tener un hijo o hija que se dedique profesionalmente al deporte: 3 de cada 4 padres (el 75\%), según datos de la EHDE (CENTRO DE INVESTIGACIONES SOCIOLÓGICAS, 2010).

\section{CONSECUENCIAS DE LA DEMOCRATIZACIÓN IMPERFECTA DEL DEPORTE}

Desde el enfoque aplicado, argüimos que ese recorrido histórico experimentado en la democratización del deporte en España, y muy especialmente lo ocurrido en el último periodo, ha desdoblado la realidad deportiva, generando dos mundos paralelos: el deporte de competición y de espectáculo y el deporte para todos. No obstante, los datos parecen evidenciar que hay un claro dominio del primero sobre el último, y ello produce ante todo la disuasión y el agravio para el deporte para todos.

De acuerdo con lo anterior, al predominar en la esfera pública del deporte la presencia mediática del espectáculo, el elitismo y la competición, podría entenderse a tenor de los datos manejados que lo que se produce es un desaliento ciudadano hacia su práctica. Tiene lugar porque la ciudadanía no persigue intereses de competición con la práctica deportiva, por lo que siente hartazgo y crítica social hacia las instituciones públicas y los grandes beneficiarios (los mass media) de un modelo que le otorga exclusividad y protagonismo a los valores derivados de ello, tal como muestran los siguientes relatos derivados del trabajo de Moscoso et al. (2013, p. 264).

A mí me gusta el fútbol y estoy cansada de poner los deportes y que solo se oiga Cristiano Ronaldo, Messi. Mira, acabas diciendo: jvale, yo no quiero más! (Mujer, 18 años, practica deporte)

Barça, Madrid, fútbol y ya está, pero a nivel de superdeportistas... Yo lo veo más en el tema de información para nosotras, para la gente normal: deporte base, que no hay nada.(Mujer, 25 años, no practica deporte)

Para mí los medios de comunicación hablan de todo menos de fútbol. O sea, lo que es el fútbol en esencia, que era a lo que yo jugaba de pequeño no tiene nada que ver con lo que se ve en la tele ni en lo que se practica ahora. Para mí, ese deporte ha evolucionado y ha cambiado para peor (...) Cuando empecé a jugar pues era un deporte que era guay, era limpio y sano y deportivo y lo que sale en las noticias es de todo menos fútbol. (Hombre, 23 años, practica deporte) 
Estos relatos parecen coincidir con los datos obtenidos a través de otras fuentes. En efecto, sobre este asunto también se le ha consultado a la ciudadanía española en la EHDE (CENTRO DE INVESTIGACIONES SOCIOLÓGICAS, 2010). Ante la pregunta "¿qué tipo de deporte piensan que está más desarrollado y recibe mayor apoyo oficial en España (el deporte espectáculo y profesional, el deporte federado o el deporte para todos)?", la mayoría manifiesta que el tipo de deporte que cuenta con mayor desarrollo y apoyo oficial en España es el deporte espectáculo. Y, a la inversa, el tipo de deporte que en opinión de la mayoría está menos desarrollado y que cuenta con menor apoyo oficial es el deporte para todos. Por último, cuando se le pregunta a la ciudadanía ¿cuál debería apoyarse más y cuál es el tipo de deporte que más le interesa?, la respuesta es contundente: la mayoría comparte que el deporte para todos es el que más apoyo debería recibir (figura 5).

Figura 5: Opinión de la ciudadanía española sobre el deporte espectáculo, el deporte federado y el deporte para todos.

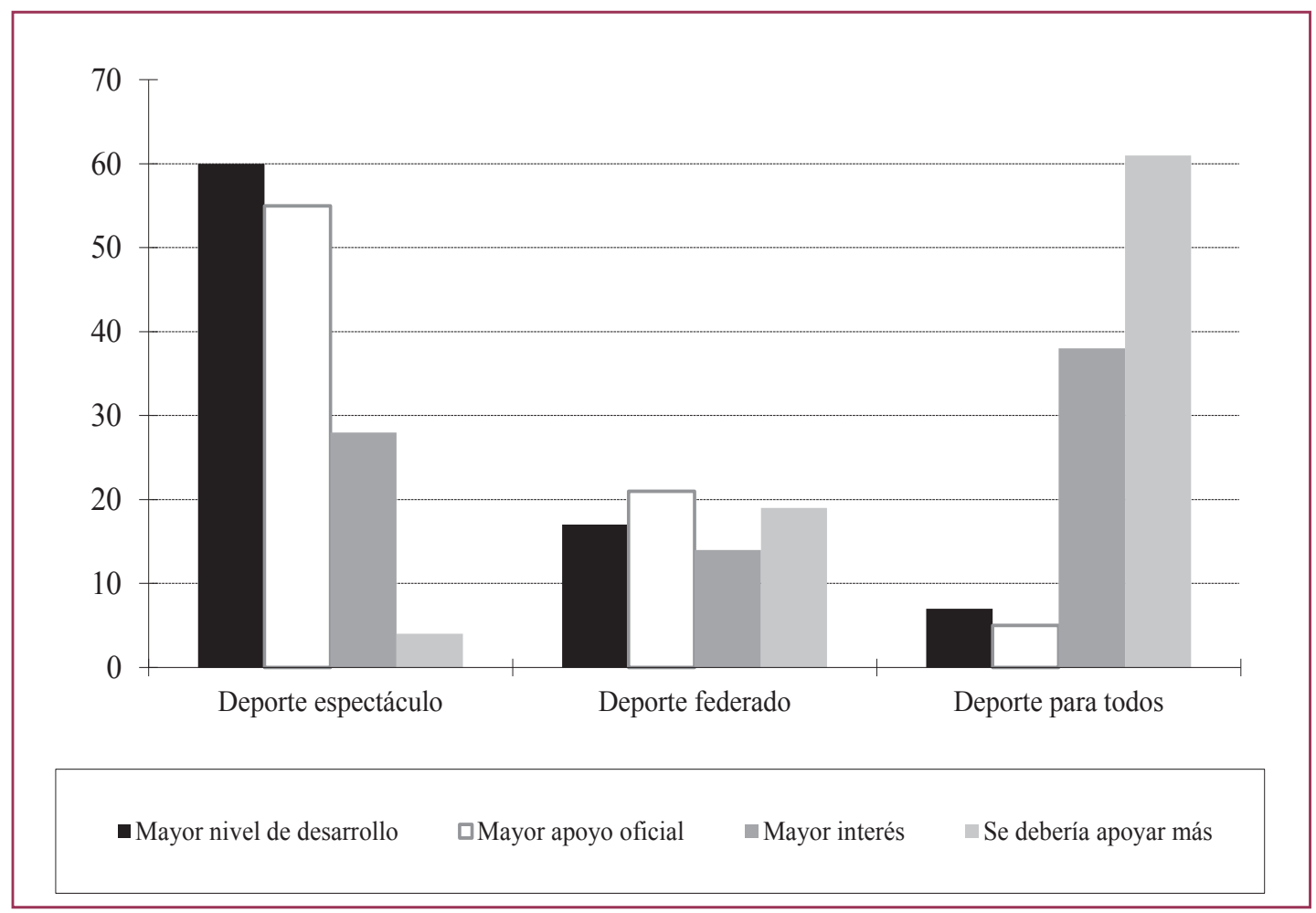

Fuente: CENTRO DE INVESTIGACIONES SOCIOLÓGICAS (2010)

Pese a estas evidencias, las mismas fuentes también muestran esa realidad ambivalente que reside en el propio sistema de valores culturales, que se apuntaba al comienzo del trabajo, a saber: la contradicción o inconsistencia de este posicionamiento ciudadano. Por un lado, el 55\% piensa que el deporte de espectáculo es el tipo de deporte que mayor apoyo recibe, al tiempo que el $61 \%$ piensa que el deporte para todos debería ser el más apoyado. Pero, por otro lado, tal como se ha señalado, el $75 \%$ quisiera que alguno de sus hijos fuera deportista profesional, el $86 \%$ admite disponer de material deportivo en su hogar - pese a que sólo el $40 \%$ practica deporte -, el $67 \%$ y el $68 \%$ admite ver por televisión retransmisiones de fútbol o de otros deportes, respectivamente, y el $47 \%$ reconoce haber asistido como espectador a algún evento o espectáculo deportivo en el último año (CENTRO DE INVESTIGACIONES SOCIOLÓGICAS, 2010). 


\section{CONCLUSIONES}

Este trabajo ha pretendido probar la situación ambivalente del sistema deportivo actual, en el contexto de la crisis del Estado de Bienestar y hegemonía de los mercados. Para ello, nos hemos centrado en reconstruir el recorrido de la democratización - a nuestro juicio imperfecta - del deporte en España. Nuestro análisis muestra una realidad que sustenta dos mundos deportivos paralelos y contradictorios.

El primer mundo es el del deporte visible, confeccionado por periodistas, publicistas, empresarios, directivos de clubes y federaciones deportivas. En él se negocia la experiencia del deporte a través de la pantalla del televisor, la marca de una prenda deportiva o el anuncio de una compañía telefónica. Es el deporte que todos conocemos, la imagen pública del deporte: la de los ídolos mediáticos, la que marca las formas, la que cuenta con el respaldo institucional. El segundo de los mundos, el del deporte invisible, es el de la mayoría ciudadana. Se trata de la experiencia real, la de los escolares y adolescentes que juegan en el patio del colegio 0 el instituto, la de las carreras populares y las actividades de los clubes en fines de semana. No entra en las formas que impone el deporte visible, no bate marcas, no obtiene victorias y, por tanto, no existe como "mirada pública" del deporte.

Los datos manejados ponen de relieve que, ante la política deportiva existente en España, y ante la posición de los mass media, las marcas deportivas y las federaciones deportivas, la ciudadanía se significa por el deporte para todos y el resto de actores por el mercado del deporte. Mientras que los recursos destinados al deporte de espectáculo han crecido substancialmente en las dos últimas décadas, la ciudadanía española, de alguna manera, no se identifica con esa política, y se siente descontenta con sus administraciones en el plano deportivo.

Un análisis crítico más reflexivo también muestra que no toda explicación ha de recaer sobre la estructura - en el sentido marxista del término -, pues la ciudadanía, como agente social, ha asumido una posición acrítica sobre esta ideología que ha imperado en el ámbito del deporte; quizá algo común en el marco de las contradicciones culturales a las que aludía Bell (1977). Desde esta perspectiva, ha de reflexionarse igualmente sobre la posibilidad de que el predominio del deporte como escenario de pasatiempo y disfrute al que nos hemos referido, con todos los agravios que puede generar para otros intereses de nuestra sociedad, no deja de sustentarse sobre comportamientos sociales que, más allá de neutralizarla, alimentan su continuidad.

Para terminar, estamos convencidos de que las ciencias sociales han de asumir un mayor compromiso público con el deporte. Ello con el fin de neutralizar la hegemonía de un modelo deportivo basado exclusivamente en el mercado. El código deontológico de ciencias como la sociología exige la provisión de argumentos críticos y estrategias que contribuyan a la defensa de los intereses generales de nuestra sociedad. Un importante grupo de sociólogos del entorno de la American Sociological Association, entre los que destaca Burawoy (2004 y 2005), han expresado argumentos en esta línea desde los años noventa. Pese a ello, en este tiempo también se ha experimentado en las ciencias sociales una dinámica de investigación sumida en el oportunismo, dejándose llevar por los temas de interés financiados por el sector privado (MOSCOSO, 2006). Ello ha dado lugar a que en muchas ocasiones quedaran relegados los verdaderos problemas sociales relacionados con el deporte. Sólo abordándolos desde la sociología pública existe alguna oportunidad de revertir algunos de los cambios de nuestra Era que suponen graves perjuicios para la sostenibilidad de la sociedad del bienestar. En el caso 
del deporte, su conversión en producto y servicio preeminentemente del mercado nos emplaza a épocas pretéritas en las que los derechos civiles carecían de sentido para las agencias de poder. Desde esta perspectiva, el reto para los científicos sociales en este siglo que avanza es preocuparse por ejercer influencia entre las grandes agencias - en los términos de Giddens (1995) - , cuyas decisiones repercuten en el sistema deportivo, para defender la pervivencia del deporte para todos como derecho ciudadano y como interés general.

\section{REFERENCIAS}

BECK, U.; GIDDENS, A.; LASH, S. Modernización reflexiva: Política, tradición y estética en el orden social moderno. Madrid: Alianza Editorial, 1997.

BELL, D. Las contradicciones culturales del capitalismo. Madrid: Alianza Editorial, 1977.

BROHM, J. M. Sociologie politique du sport. París: Jean Pierre-Legardé, 1982.

BURAWOY, M. The world needs public sociology. Sosiologisk tidsskrift, Langnesgrunnen, v. 12, n. 3, p. 255-272, 2004.

BURAWOY, M. For public sociology. American Sociological Review, Washington, v. 70, p. 4-28. 2005.

COMISIÓN EUROPEA. Carta Europea del Deporte para Todos. Resolución 76/41. Bruselas: Comisión Europea, 1976.

COMISIÓN EUROPEA. Carta Europea del Deporte. Resolución 92/3. Bruselas: Comisión Europea, 1992.

COMISIÓN EUROPEA. Libro Blanco sobre el Deporte. COM (2007) 391. Bruselas: Comisión Europea, 2007.

CENTRO DE INVESTIGACIONES SOCIOLÓGICAS (CIS). Hábitos Deportivos en España I, Estudio n‥ 2198. Madrid: CIS, 1995.

CENTRO DE INVESTIGACIONES SOCIOLÓGICAS (CIS). Hábitos Deportivos en España III, Estudio n‥ 2599. Madrid: CIS, 2005.

CENTRO DE INVESTIGACIONES SOCIOLÓGICAS (CIS). Hábitos Deportivos en España IV, Estudio no. 2833. Madrid: CIS, 2010.

CONSEJO SUPERIOR DE DEPORTES (CSD). II Censo Nacional de Instalaciones Deportivas. Madrid: CSD, 1997.

CONSEJO SUPERIOR DE DEPORTES (CSD). III Censo Nacional de Instalaciones Deportivas 2005. Madrid: CIS, 2005.

DESBORDES, M; OHL, F; TRIBOU, G. Estrategias del márketing deportivo: Análisis del consumo deportivo. Barcelona: Paidotribo, 1999.

ESPAÑA. Ministerio de la Presidencia. Ley 13/80, de 31 de marzo, General de la Cultura Física y del Deporte. Boletín Oficial del Estado, Madrid, n. 89, de 12 de abr. de 1980, p. 7908- 7913.

ESPAÑA. Constitución Española de 1978. Disponible en: <http://www.congreso.es/consti/ constitucion/indice/index.htm>. Acceso en: 30 abril 2014. 
GARCÍA, M; LAGARDERA, F. La perspectiva sociológica del deporte. En: GARCÍA, M; PUIG, N; LAGARDERA, F. (Ed.). Sociología del Deporte. Madrid: Alianza Editorial, 1998. p. 13-37.

GARCÍA, J; ALCOLEA, J. Los derechos audiovisuales de retransmisiones deportivas y su repercusión en el mercado televisivo español. Razón y Palabra, Atizapán de Zaragoza, v. 77, 2011.

GARCIA FERRANDO, M. Los españoles y el deporte, 1980-1996. Un estudio sociológico sobre comportamientos, actitudes y valores. Valencia: Tirant lo Blanch/Consejo Superior de Deportes, 1997.

GIDDENS, A. La constitución de la sociedad: bases para la teoría de la estructuración. Buenos Aires: Amorrortu, 1995.

GIL-CALVO, E. Modernización y Cambio Político. En: BENEDICTO, J; MORÁN, M.L. (Ed.). Sociedad y Política. Madrid: Alianza Universidad, 1995. p. 327-368.

INGLEHART, R. El cambio cultural en las sociedades industriales avanzadas. Madrid: Centro de Investigaciones Sociológicas, 1991.

INSTITUTO NACIONAL DE ESTADÍSTICA. Estadística de sociedades y clubes federados.

Disponible en: $<$ http://www.ine.es/jaxi/menu.do?type=pcaxis\&path=/t12/a115/a01/\&file=pcaxis $>$. Acceso en: 30 abril 2014

LAGARDERA, F. Deporte y calidad de vida. La sociedad deportivizada. En: GARCÍA, C. (coord.). Actas del 8 Congreso Científico Olímpico 1992. Málaga: Instituto Andaluz del Deporte, 1992. p. $412-423$

MOSCOSO, D. La sociología del deporte en España: estado de la cuestión. Revista Internacional de Sociología, Córdoba, n. 44, p. 177-204, 2006.

MOSCOSO, D. La brecha deportiva y el sueño olímpico. Disponible en: <http://elpais.com/ elpais/2013/09/16/opinion/1379343283_113729.html>. Acceso en: 29 diciembre 2013.

MOSCOSO, D.; MUÑOZ, V. Deporte, inclusión y diversidad social. Anduli. Revista Andaluza de Ciencias Sociales, Sevilla, n. 11, p. 13-19, 2012.

MOSCOSO, D; MARTIN, M; SÁNCHEZ, R; PEDRAJAS, N. Sedentarismo activo. Ocio, actividad física y estilos de vida de la juventud española. Archivos de Medicina del Deporte, Madrid, v. 30, n. 6, p. 260-270. 2013.

ROBERTSON, R; GIULIANOTTI, R. Fútbol, globalización y glocalización. Revista Internacional de Sociología, Madrid, n. 45, p. 9-35, 2006.

RODRÍGUEZ, A. El deporte en la construcción del espacio social. Madrid: Centro de Investigaciones Sociológicas, 2008. 


\section{Endereço para correspondência:}

Pablo de Olavide University

Carretera Utrera, km 1, s/n - Edificio 11, 4ª Planta, Despacho 18 - 41013 Sevilla - Spain.

\section{Apoyo financiero:}

Convocatoria de Estudios Sociales de la Fundación Obra Social de La Caixa y del Consejo Superior de Deportes del Gobierno de España . 